\title{
Determinación de micotoxinas presentes en alimentos de consumo infantil comercializados en México
}

\section{Determination of mycotoxins present in foods for infant consumption marketed in Mexico}

DOI: $10.46932 / \mathrm{sfjdv2n4-013}$

Received in: May 1st, 2021

Accepted in: Jun 30th, 2021

\section{Reyes Pérez Jesica Abigali}

Ingeniera agrícola. Graduada (Sin institución actual). UNAM, Facultad de Estudios Superiores Cuautitlán (Campo 4), Departamento de Ingeniería Agrícola, Carr. Cuautitlán-Teoloyucan Km. 2.5, San Sebastián Xhala, 54714 Cuautitlán Izcalli, Edo. de Méx.

E-mail: abi.rpz08@gmail.com

\section{Quezada Viay Martha Yoland}

Doctorado En Parasitología Agrícola, Maestría en Ciencias Bioquímicas. UNAM-FESC-Unidad de Investigación en Granos y Semillas UNIGRAS, Av. Dr. Jiménez Cantú S/N Col. Atlamica, CP 54729, Cuautitlán Izcalli, Edo. de Méx.

E-mail: yolaqviay@gmail.com

\section{Moreno Lara Josefina}

Maestría en microbiología. UNAM-FESC-Unidad de Investigación en Granos y Semillas UNIGRAS, Av. Dr. Jiménez Cantú S/N Col. Atlamica, CP 54729, Cuautitlán Izcalli, Edo. de Méx.

E-mail: joslara2004@yahoo.com.mx

\section{Torres Flores David}

Ingeniero Agrícola. Graduado (Sin institución actual). UNAM, Facultad de Estudios Superiores Cuautitlán (Campo 4), Departamento de Ingeniería Agrícola, Carr. Cuautitlán-Teoloyucan Km. 2.5, San Sebastián Xhala, 54714 Cuautitlán Izcalli, Edo. de Méx.

E-mail: davidtorresflores9@gmail.com

\section{Andrea Alejandra Arrúa Alvarenga}

Doctorado en Ciencias en Parasitología Agrícola. Universidad Nacional de Asunción, Centro Multidisciplinario de Investigaciones Tecnológicas, San Lorenzo Paraguay. Dr. Gaspar Villamayor c/ Dr. Cecilio Baéz, Campus Universitario de la UNA, San Lorenzo

E-mail: aaarrua@gmail.com

\section{RESUMEN}

Los cereales y sus subproductos son la base de la alimentación en México y en países en desarrollo. El cereal seco es un alimento basado en granos de cereales y/o leguminosas que se elaboran para lograr un escaso contenido de humedad y se fragmentan para permitir su dilución con agua, leche y otro líquido conveniente para niños y lactantes. Los efectos adversos de las micotoxinas incluyen problemas en el crecimiento infantil, defectos en el desarrollo del tubo neural, daños al sistema inmunológico, enfermedades renales, y mayores probabilidades de desarrollar cáncer de hígado y esófago. Por lo cual, su estudio es de gran importancia en lo que respecta a la salud pública. Las aflatoxinas son carcinógenos potentes que pueden afectar especialmente al hígado y al riñón; son causa de cáncer hepático y se han relacionado con otros tipos de cáncer. El presente trabajo consistió en determinar la cantidad de 
aflatoxinas (AF) totales en alimentos de consumo infantil, extrayéndose de éstos mediante columnas de anticuerpos monoclonales para conocer sus niveles de concentración con el método "Aflatest - Vicam". Las muestras analizadas fueron principalmente cereales para consumo infantil entre otros productos como avenas, galletas y frituras. Los resultados reportaron que el $83.3 \%$ de los productos analizados se encontraban libres de aflatoxinas, mientras que el $16.7 \%$ arrojaron concentraciones mínimas que no rebasaron los límites máximos permisibles por la NOM-187-SSAI/SCFI-2002, la cual establece $20 \mu \mathrm{g} / \mathrm{kg}$ de concentración máxima en harinas y cereales.

Palabras clave: Micotoxinas, Alimentación infantil, Aflatoxinas, Cereales, Salud pública, Aspergillus flavus.

\begin{abstract}
Cereals and their by-products are the basis of food in Mexico and developing countries. Dry cereal is a food based on grains of cereals and/or leguminous plants which are made to achieve a low moisture content and are fragmented to allow dilution with water, milk and other liquid liquid suitable for infants and infants. Adverse effects of mycotoxins include problems in childhood growth, defects in the development of the neuronal tube, damage to the immune system kidney diseases, and more likely to develop liver cancer and esophageal cancer. Therefore, his study is of great importance in regards to public health. Aflatoxins are potent carcinogens that can affect any organ or system, and especially to the liver and kidney; they cause liver cancer and have been linked to other types of cancer. The present work consisted of determining the amount of total aflatoxins (AF) in infant foods by monoclonal antibodies to know their concentration levels by the "aflatest - vicam" method, the samples analysed were mainly cereals for infant consumption among other products such as oats, biscuits and fritters. The results reported that $83.3 \%$ of the products analysed were free of aflatoxins, while $16.7 \%$ reported minimal concentrations, thus not exceeding the maximum permissible concentrations of the NOM-187-SSAI/SCFI-2002 which sets $20 \mu \mathrm{g} / \mathrm{kg}$ maximum concentration in flour and cereals.
\end{abstract}

Key words: Mycotoxin, Infant Feeding, Aflatoxins, Cereals, Public Health, Aspergillus flavus.

\title{
1 INTRODUCCIÓN
}

Los hongos producen una gran variedad de compuestos tóxicos, conocidos como micotoxinas. Micotoxina, deriva de las palabras griegas: mikes y toxina, que significa hongo y veneno respectivamente. Algunos hongos, durante su fase estacionaria, son capaces de producir metabolitos secundarios tóxicos, que tienen efectos nocivos en la salud humana y animal. (Fokunang et al., 2006; Soriano, 2007). Dado su carácter inevitable e imprevisible, la contaminación por micotoxinas plantea un problema especial para la inocuidad de los alimentos (López-García et al., 1999).

La contaminación con micotoxinas en los granos generalmente es un proceso; puede iniciarse en el campo, aumentar durante la cosecha y operaciones de secado y continuar acumulándose durante el almacenamiento (Bogantes et al., 2004).

Las micotoxinas son compuestos químicos producidos de forma natural por una serie de hongos, principalmente, Aspergillus, Penicillium, Fusarium y Alternaria. Dichos hongos atacan a cultivos en campo, principalmente a forrajes, cereales, leguminosas, frutos secos, frutas y hortalizas. 
Las micotoxinas, al ser compuestos termoestables y resistentes, persisten tras los procesos de secado, molienda y procesado de los alimentos procedentes de los cultivos, entrando así en la cadena alimentaria.

Según la Organización de las Naciones Unidas para la Alimentación (FAO), el 25\% de los cultivos alimentarios mundiales se ven afectados por hongos productores de micotoxinas. Las estimaciones de las pérdidas mundiales de productos alimenticios debidas a las micotoxinas son del orden de 1.000 millones de toneladas al año (FAO, 2019).

Las micotoxinas no solo representan un riesgo para la salud humana y animal, sino que también afectan la seguridad alimentaria y la nutrición al reducir el acceso de las personas a alimentos saludables. La Organización Mundial de la Salud (OMS) exhorta a las autoridades nacionales a supervisar y garantizar que los niveles de micotoxinas en los alimentos que se comercializan en sus países sean lo más bajos posible y cumplan con los niveles máximos, las condiciones y las legislaciones nacionales e internacionales (OMS, 2018).

\section{MICOTOXINAS DE GRAN IMPORTANCIA EN LA SALUD PÚBLICA}

Las micotoxinas contaminan principalmente cereales, los cuales se infectan con el hongo antes y durante la cosecha o el almacenamiento. Actualmente, más de 400 toxinas producidas por 350 especies de hongos han sido aisladas y caracterizadas; de éstas, las investigaciones se han enfocado en aquellas que causan daños significativos a humanos y animales (Brase et al., 2009). Ejemplos de micotoxinas de gran importancia en salud pública incluyen aflatoxinas, tricotecenos, fumonisinas, ocratoxinas y zearalenona (Abrunhosa et al., 2014). Los efectos de dichas toxinas cuestan millones de dólares anualmente en pérdidas a nivel mundial en salud humana, animal y productos agrícolas (Vasanthi y Bhat, 1998).

Al consumir alimentos contaminados con micotoxinas, se producen en las personas y en los animales una serie de efectos tóxicos que dependen básicamente de la propia toxicidad de la/s micotoxina/s presente/s en el alimento consumido.

\section{AFLATOXINAS}

Las aflatoxinas son un grupo de aproximadamente 20 compuestos, producidos por especies del género Aspergillus. El término "aflatoxina" fue acuñado en Inglaterra en la década de 1960, cuando miles de pavos fueron alimentados con harina de cacahuate contaminada con la micotoxina murieron a causa de una enfermedad desconocida que se denominó enfermedad "X" de los pavos (Blount, 1961). 
Posteriormente se confirmó la presencia de una toxina del hongo Aspergillus flavus en el extracto del medio de crecimiento, la cual mostró toxicidad en ratas y patos (Lancaster et al., 1961).

Las aflatoxinas contaminan cultivos básicos para la alimentación, incluyendo el maíz, el cacahuate y la nuez, causando trastornos agudos y crónicos sobre la población humana.

Hay varios tipos naturales de aflatoxinas (14 o más) particularmente peligrosas para los humanos y los animales, dado que se han encontrado en los principales cultivos de alimentos. Sin embargo, la mayor parte de la exposición humana procede de frutos secos y cereales contaminados, así como de productos derivados de ellos (OMS, 2018).

Existen cuatro aflatoxinas principales, conocidas como aflatoxina B1, aflatoxina B2, aflatoxina G1 y aflatoxina G2. La letra B indica que estas aflatoxinas tienen fluorescencia azul (blue) frente a la luz ultravioleta (365 nm), mientras que la letra $\mathrm{G}$ indica la fluorescencia verde (green). La aflatoxina B1 es la más tóxica de todas, y se ha correlacionado con el carcinoma hepatocelular en humanos y en una amplia variedad de especies animales (Richard, 2007; Wu et al., 2014).

En colaboración con la FAO, la Organización Mundial de la Salud (OMS) evalúa los datos científicos y los riesgos para definir niveles de exposición seguros. A partir de estas evaluaciones del riesgo, se recomiendan concentraciones máximas permisibles de aflatoxinas en diferentes alimentos, valores sobre los que asientan las reglamentaciones nacionales para limitar la contaminación.

En lo que se refiere a las aflatoxinas en humanos, se ha observado una correlación entre el consumo de alimento contaminado con estas toxinas y el desarrollo de cáncer de hígado. Por tal motivo, la Organización Mundial de la Salud (OMS) en conjunto con la Agencia Internacional para la Investigación en Cáncer (WHO-IARC, por sus siglas en inglés) han evaluado los efectos de las aflatoxinas, y para 1993, las clasificaron en grupos, destacando al grupo 1 como una mezcla que ocurre naturalmente y que probablemente favorece el desarrollo del cáncer de hígado (WHO-IARC, 1993). Además, existen casos de aflatoxicosis agudas con manifestaciones clínicas que incluyen vómito, dolor abdominal, edema pulmonar e infiltración de grasa (Wu et al., 2014).

La exposición a aflatoxinas (dosis-dependiente) también ha sido relacionada con el retraso en el crecimiento infantil, una condición en la cual la altura de los niños está por debajo de la referencia de crecimiento establecida por la Organización Mundial de la Salud (WHO-IARC, 1993). Este tipo de estudio es importante desde el punto de vista de salud pública, debido a que se ha asociado la intoxicación infantil con vulnerabilidad a enfermedades infecciosas y deficiencias en el aprendizaje (Khlangwiset et al., 2011). Además, se ha demostrado que la ingesta de alimentos contaminados con aflatoxinas en mujeres en etapa de lactancia condujo a un menor peso y talla de los bebés lactantes (Mahdavi et al., 2010) 


\section{TRANSMISIÓN EN LA CADENA ALIMENTARIA}

Las micotoxinas pueden entrar en la cadena alimentaria por las siguientes vías (Asociación española

de

fabricantes

de

cereales,

2011):

- Directamente a través de los alimentos sin procesar susceptibles de la contaminación por micotoxinas son: cereales, legumbres, semillas oleaginosas, frutas, hortalizas, frutos secos, frutas desecadas, café, cacao y especias.

- Directamente por los alimentos procesados que pueden contribuir a la exposición de micotoxinas, al no destruirse durante el procesado son: productos y derivados a base de cereales (pan, pasta, cereales de desayuno, etc.), bebidas (vino, café, cacao, cerveza, zumos), y los alimentos infantiles. Dentro de los alimentos infantiles se encuentran los cereales de desayuno cuya cadena alimentaria comienza con la producción, recolección y almacenamiento de materias primas y continúa con los procesos de elaboración, envasado, distribución, venta y preparación en el hogar por el consumidor hasta su ingesta.

Las materias primas más utilizadas son: trigo, maíz, arroz, avena, cebada y otros cereales comestibles, ya sean enteros o troceados. Además de los cereales, en ocasiones se utilizan otros ingredientes como cacao, frutas, frutos secos, malta, miel y leche. Los cereales más relevantes desde el punto de vista de la nutrición humana son el trigo y el arroz, que constituyen la base de la alimentación de cuatro quintas partes de la población mundial (Asociación española de fabricantes de cereales, 2011).

\section{LEGISLACIÓN Y CANTIDADES PERMISIBLES DE AFLATOXINAS}

Desde su descubrimiento en la década de 1960, las aflatoxinas han sido objeto de varias evaluaciones toxicológicas y de la exposición a través de los alimentos por parte del Comité Mixto FAO/OMS de Expertos en Aditivos Alimentarios. Dichas evaluaciones son utilizadas por la Comisión del Codex Alimentarius, que desde 1963 viene trabajando en la elaboración de normas internacionales armonizadas sobre los alimentos con el fin de proteger la salud de los consumidores y garantizar prácticas comerciales equitativas.

Como medidas de gestión del riesgo en el caso de las aflatoxinas, además del establecimiento de límites máximos en la legislación, existen varios Códigos de Prácticas de higiene a nivel internacional en el Codex Alimentarius que ayudan a disminuir la presencia de los hongos productores de aflatoxinas, y por tanto, a reducir el nivel de las mismas en determinados alimentos: Las normas del Codex establecen las concentraciones máximas de contaminantes y toxinas naturales, como las aflatoxinas, que pueden 
contener los alimentos, y son la referencia para el comercio internacional de alimentos, de modo que todos los consumidores puedan confiar en que los alimentos que compran cumplen las normas de inocuidad y calidad acordadas, independientemente de su lugar de producción. (OMS, 2018)

Diferentes legislaciones tanto internacionales como nacionales establecen límites máximos de tolerancia en diferentes alimentos, además de normalizar muestreos y análisis, esto debido a que el contenido de sustancias tóxicas es inevitable en los alimentos. En México existe la norma oficial Mexicana NOM 188-SSA1-2002 y la NOM-247-SSA1_2008, para cereales y sus productos, donde establecen un límite máximo de aflatoxinas totales de $20 \mu \mathrm{g} / \mathrm{kg}$. La unión europea establece un límite máximo de 2 $\mu \mathrm{g} / \mathrm{kg}$ de aflatoxinas totales en cereales y derivados. En América Latina y MERCOSUR (Brasil, Paraguay, Uruguay y Argentina) establecen un límite de aflatoxinas totales de $20 \mu \mathrm{g} / \mathrm{kg}$, al igual que Estados Unidos (Vega, 2012) (FAO, 2004).

Por lo mencionado anteriormente el objetivo de este trabajo fue determinar la cantidad de aflatoxinas (AF) totales en alimentos de consumo infantil en México mediante anticuerpos monoclonales para conocer sus niveles de concentración.

\section{METODOLOGÍA}

\subsection{SELECCIÓN DE MUESTRAS}

Se seleccionaron en diferentes establecimientos 24 productos dirigidos principalmente para el consumo infantil, de ellos se realizó un listado del nombre y tipo del producto, contenido de ingredientes, fecha de caducidad y número de lote al que pertenecieron.

Tabla 3: Listado de productos evaluados en su concentración de AF

\begin{tabular}{|l|l|}
\hline Productos & Cereal de miel \\
\hline Hojuelas de maíz azucaradas & Hojuelas de avena \\
\hline Cereal de trigo inflado y endulzado sabor vainilla & Avena instantánea integral con sabor miel con nuez \\
\hline Arroz inflado sabor chocolate $*$ & Avena instantánea integral con manzana \\
\hline Aritos de maíz, trigo y avena $*$ & Fritura de maíz con sal de mar y limón \\
\hline Hojuelas de maíz con azúcar $*$ & Hojuelas de maíz cubiertas con chocolate \\
\hline Bolitas de maíz y arroz sabor chocolate $*$ & Galletas de animalitos \\
\hline Hojuelas de maíz & Puré de manzana, plátano, pera y mango \\
\hline Hojuelas de maíz con azúcar & Arroz inflado sabor chocolate \\
\hline Hojuelas de maíz & Aritos de maíz, trigo y avena \\
\hline Hojuelas de maíz sabor chocolate & \\
\hline
\end{tabular}




\begin{tabular}{|l|l|}
\hline Hojuelas de maíz azucaradas & Bolitas de maíz y arroz sabor chocolate \\
\hline $\begin{array}{l}\text { Cereal sabor manzana, hojuelas de avena, trigo, pasas y } \\
\text { manzana deshidratada }\end{array}$ & Hojuelas de maíz con azúcar \\
\hline$* 2$ muestras: Mismo producto en dos diferentes presentaciones \\
\hline
\end{tabular}

\section{CUANTIFICACIÓN DE AFLATOXINAS "AFLATEST-VICAM"}

La cuantificación total de aflatoxinas se realizó por medio del método Aflatest de Vicam (Vicam, 1999) Se pesaron 50 gr de cada muestra de cada producto y se agregó $100 \mathrm{ml}$ de metanol (80\%) junto con 5 gr de cloruro de sodio. Posteriormente se licuó por 1 minuto, se filtró con papel Whatman no.1. y posteriormente se tomaron $10 \mathrm{ml}$ del filtrado para diluirse en $40 \mathrm{ml}$ de agua destilada, se filtró la dilución con papel fibra de vidrio (figura 1) y se pasaron $10 \mathrm{ml}$ por la columna de anticuerpos monoclonales previamente acondicionada.

La misma se lavó 2 veces con agua destilada (figura 2) y posteriormente se pasó $1 \mathrm{ml}$ de metanol grado HPLC por la columna y se recuperó en un vial. Se añadió $1.0 \mathrm{ml}$ de solución reveladora de bromo, se agitó por 3 segundos y se leyó en un fluorómetro marca Vicam serie 4 (figura 3) (Moreno, 2004; NOM188-SSA1-2002).

Figura 1. Preparación de muestras, pesaje, molienda, extracción, filtración y dilución.

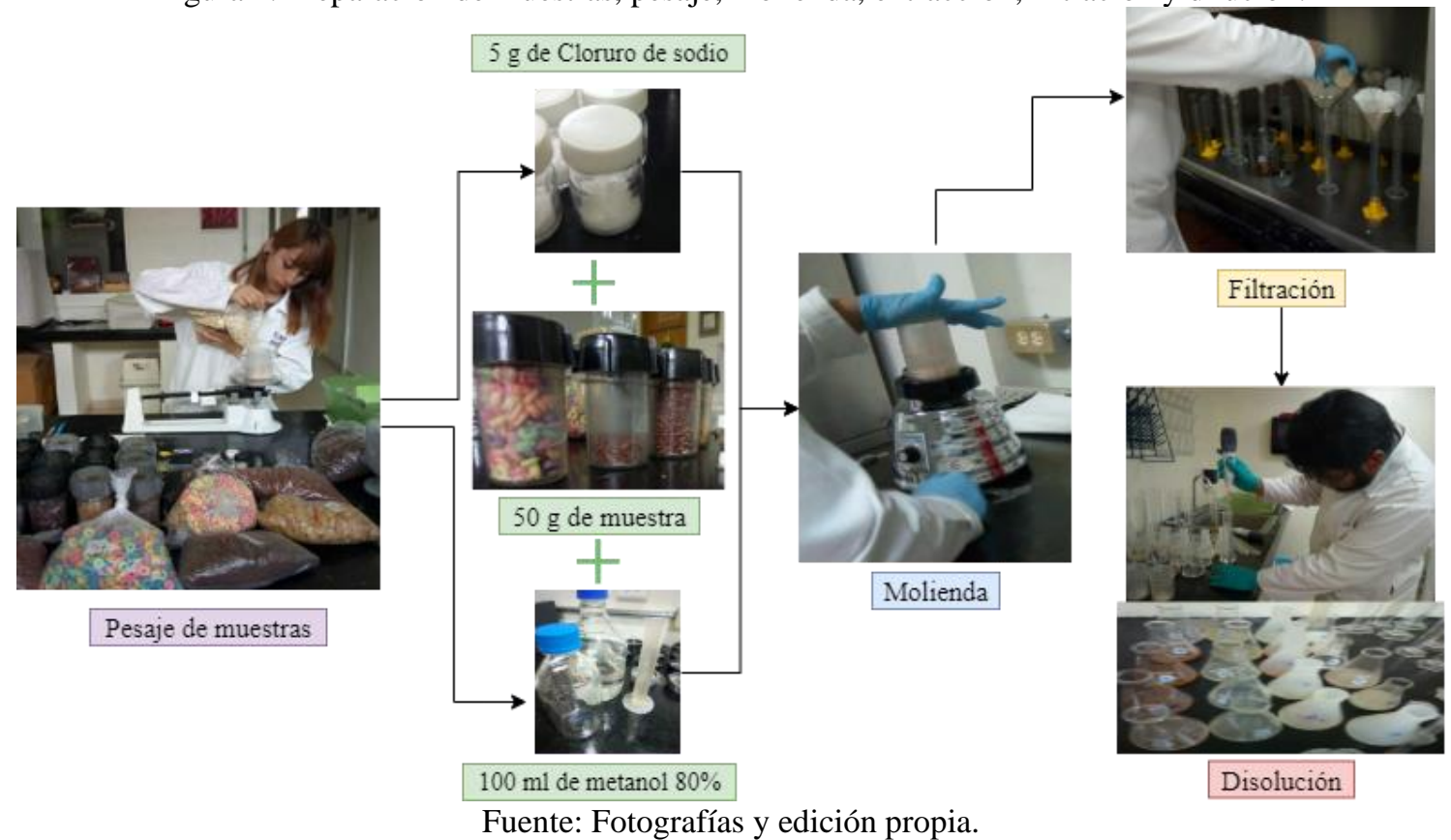


Figura 2. Purificación y extracción de AFt (Aflatoxinas totales).

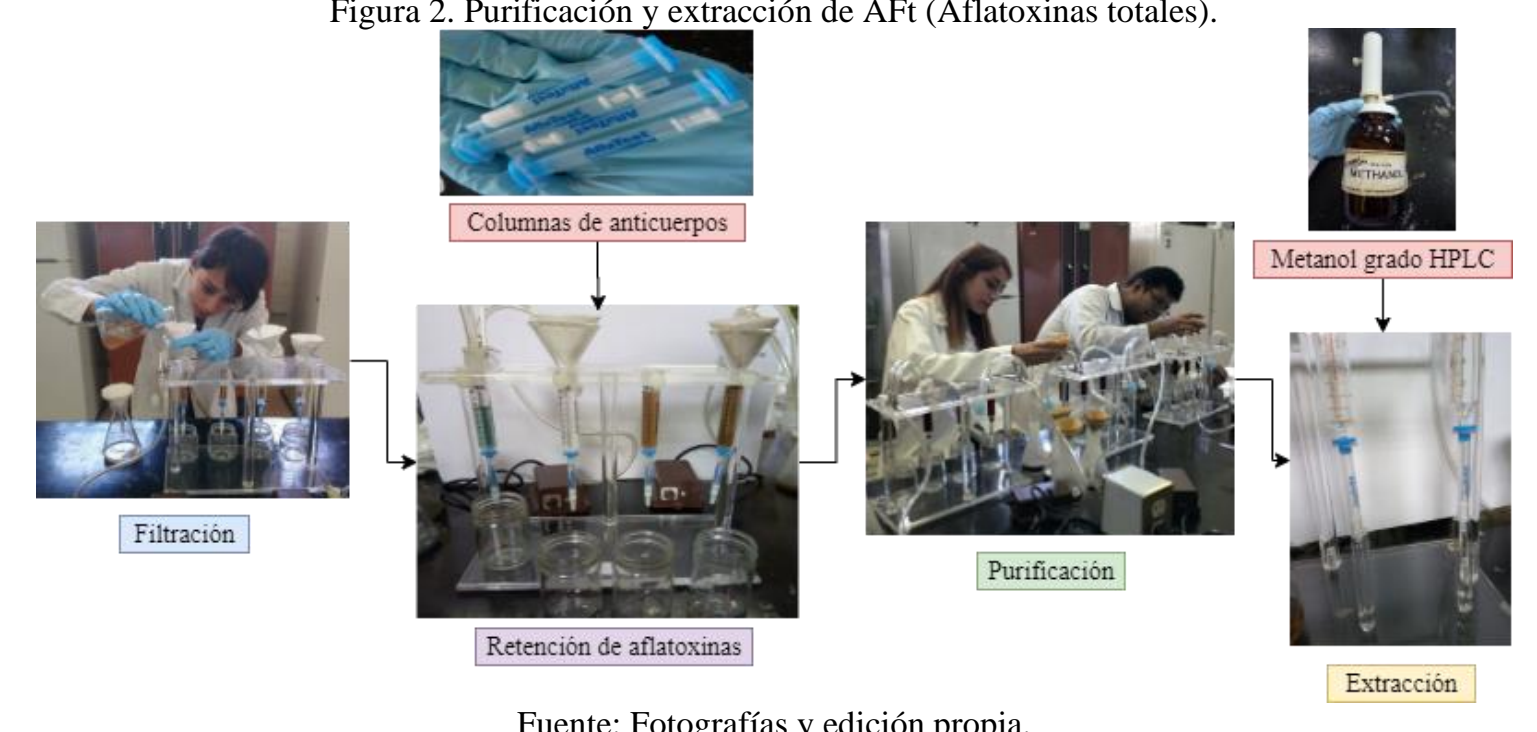

Figura 3. Amplificación de la fluorescencia y cuantificación de AFt
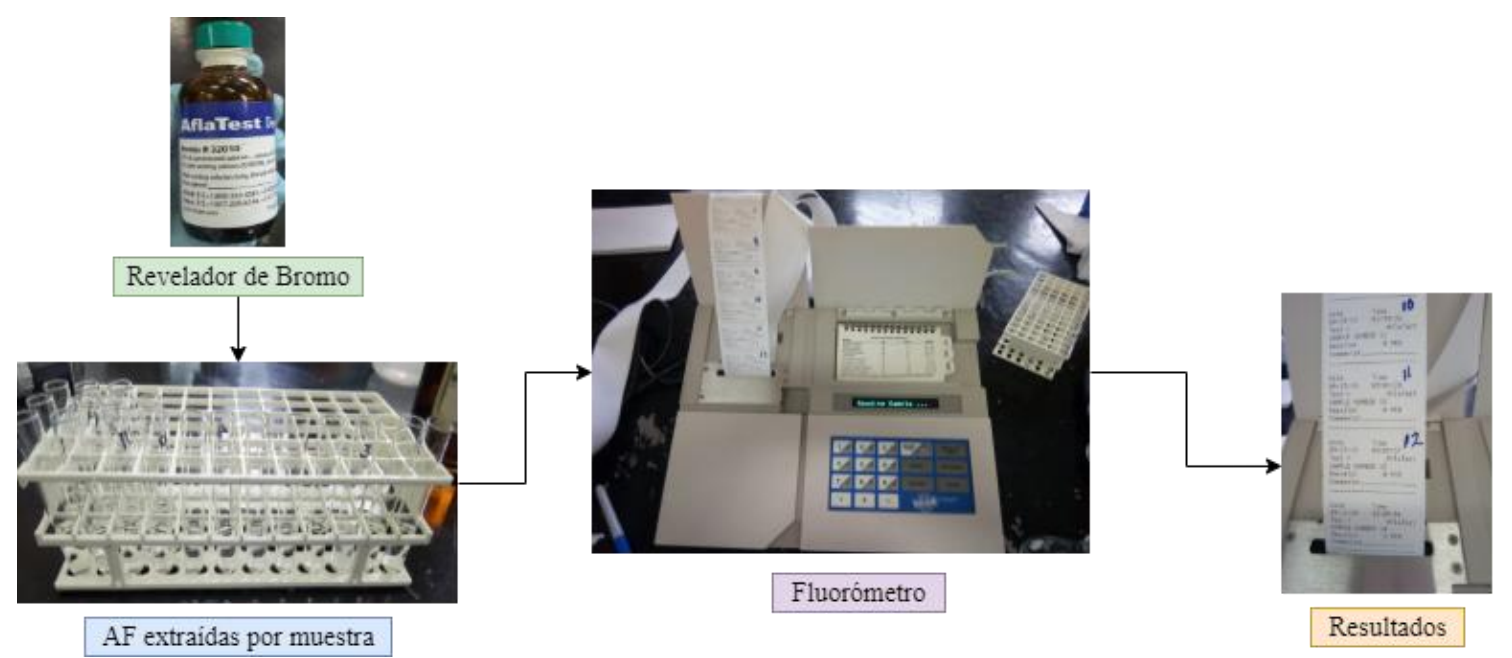

Fuente: Fotografías y edición propia.

\section{RESULTADOS}

Los resultados mostraron que el $16.7 \%$ de las muestras, presentaron niveles mínimos detectables de Aflatoxinas totales, las cuales oscilaban entre $0.53 \mu \mathrm{g} / \mathrm{kg}$ y $1.16 \mu \mathrm{g} / \mathrm{kg}$ (tabla 4 ), con una media de 0.83 $\mu \mathrm{g} / \mathrm{kg}$. El 83.3\% restante reportó resultados de $0 \mu \mathrm{g} / \mathrm{kg}$.

El nivel más alto fue hallado en una muestra de cereal de arroz inflado con sabor chocolate. 
Tabla 4. Concentración de AFt en muestras de alimentos de consumo infantil

\begin{tabular}{|l|l|l|l|l|}
\hline Producto & $\begin{array}{l}\text { Arroz inflado sabor } \\
\text { chocolate }\end{array}$ & $\begin{array}{l}\text { Hojuelas de } \\
\text { maíz } \\
\text { azúcar }\end{array}$ & $\begin{array}{l}\text { Bolitas de maíz y } \\
\text { con } \\
\text { choz } \\
\text { chocolate }\end{array}$ & $\begin{array}{l}\text { Avena instantánea } \\
\text { integral con sabor miel } \\
\text { con nuez }\end{array}$ \\
\hline AF $(\boldsymbol{\mu g} / \mathrm{kg})$ & 1.16 & 0.536 & 0.93 & 0.705 \\
\hline $\begin{array}{l}\text { Contenidos máximos permitidos } \\
\text { en México }\end{array}$ & $20 \mu \mathrm{g} / \mathrm{kg}$ & \multicolumn{5}{|l|}{} & $\checkmark$ \\
\hline $\begin{array}{l}\text { Cumplimiento con la norma } \\
\text { NOM-247-SSAI/SCFI-2008 }\end{array}$ & $\checkmark$ & $\checkmark$ & $\checkmark$ & \\
\hline
\end{tabular}

Los productos fueron agrupados de acuerdo al tipo de cereal de elaboración. Se obtuvo que el 50\% de los productos analizados fueron elaborados principalmente a base de maíz, seguidos de cereales combinados (17\%) y posteriormente productos a base de avena con un 13\%, sólo uno de los productos (puré de frutas mixtas) no perteneció a esta categoría sin embargo se consideró dentro del número de muestras.

En la tabla 5 se puede observar que el cereal libre de AF fue el trigo, donde los dos productos analizados arrojaron una concentración de $0 \mu \mathrm{g} / \mathrm{kg}$. Dentro de los cereales con presencia de AF se encontró de mayor a menor el arroz con $50 \%$, avena $33 \%$, cereales combinados con $25 \%$ y maíz con un $8 \%$.

Tabla 5: Recuento y porcentaje de productos analizados por tipo de cereal y por contenido de aflatoxinas totales

\begin{tabular}{|l|l|l|l|l|}
\hline Tipo de cereal & $\begin{array}{l}\text { Cantidad de } \\
\text { productos }\end{array}$ & $\begin{array}{l}\text { \% de productos } \\
\text { evaluados por } \\
\text { tipo de cereal }\end{array}$ & $\begin{array}{l}\text { Cantidad de } \\
\text { productos con } \\
\text { AFt }\end{array}$ & $\begin{array}{l}\text { \%Ft por tipo de cereal } \\
\text { AFt productos con }\end{array}$ \\
\hline Maíz & 12 & $50 \%$ & 1 & $8 \%$ \\
\hline Arroz & 2 & $8 \%$ & 1 & $50 \%$ \\
\hline Avena & 3 & $13 \%$ & 1 & $33 \%$ \\
\hline Trigo & 2 & $8 \%$ & 0 & 0 \\
\hline Combinados & 4 & $17 \%$ & 1 & $25 \%$ \\
\hline $\begin{array}{l}\text { Otro producto: } \\
\text { Puré }\end{array}$ & 1 & $4 \%$ & 0 & 0 \\
\hline Total & 24 & $100 \%$ & 4 & \\
\hline
\end{tabular}

\section{DISCUSIÓN}

Los resultados de la tabla 4 muestra que la incidencia de aflatoxinas en alimentos de consumo infantil comercializados en México fue de $16.7 \%$, presentando niveles detectables que oscilan entre 0.53 
$\mu \mathrm{g} / \mathrm{kg}$ y $1.16 \mu \mathrm{g} / \mathrm{kg}$. Por lo tanto, los niveles de contaminación con aflatoxinas se encuentran por debajo de lo permitido en la NOM-247-SSAI/SCFI-2008 $(20 \mu \mathrm{g} / \mathrm{kg})$ y por las legislaciones establecidas por la Unión Europea para cereales y derivados $(2 \mu \mathrm{g} / \mathrm{kg})$ y aflatoxinas B1 y totales $(4 \mu \mathrm{g} / \mathrm{kg})$, MERCOSUR $(20$ $\mu \mathrm{g} / \mathrm{kg}$ ) y Estados Unidos $(20 \mu \mathrm{g} / \mathrm{kg})$. El $83.3 \%$ de las muestras analizadas se encontraron libres de contaminación por aflatoxinas.

La contaminación por aflatoxinas en granos es un problema de importancia internacional. El progresivo aumento de las temperaturas puede conducir a un mayor predominio de especies de Aspergillus productoras de aflatoxinas como respuesta al estrés al que se ven sometidas las plantas debido a las nuevas condiciones climáticas (Gonzalo, 2018).

El cambio climático está ocasionando la modificación de las comunidades microbianas de cada zona con presencia de organismos que resisten mejor las nuevas condiciones. Esta característica resalta la necesidad de contar con políticas de inocuidad más efectivas para este tipo de productos y contaminantes.

La presencia de aflatoxinas en cuatro muestras, ponen de manifiesto que existen algunas deficiencias en el manejo postcosecha de granos especialmente durante el almacenamiento. Los resultados obtenidos, aunque son puntuales, reflejan la necesidad de implementar estrategias para la vigilancia, el seguimiento y control de este tipo de contaminantes de ocurrencia natural los cuales tienen implicaciones directas sobre la salud humana. Se requiere continuar este tipo de muestreo con el fin de obtener mayor cantidad de datos que permitan analizar el comportamiento de los niveles de aflatoxinas en alimentos de consumo humano.

En los resultados de la tabla 5 se muestra que los productos analizados y que contaron con la presencia mínima de aflatoxinas pertenecen al grupo de los cultivos básicos para la alimentación humana, siendo el maíz el de mayor importancia para México. Sin embargo, de acuerdo a la cantidad de muestras analizadas por tipo de cereal, el $50 \%$ de las muestras elaboradas con arroz contenían niveles mínimos de AF. Es posible que el porcentaje de incidencia por tipo de cereal no sea significativo, por lo que se recomienda aumentar el número de muestras analizadas en trabajos futuros, para poder afirmar la presencia de aflatoxinas en productos elaborados a base de maíz, arroz y avena. Se hace necesario continuar monitoreando en diferentes puntos de comercialización y por mayores periodos de tiempo.

Los resultados obtenidos sugieren que los cereales analizados que se comercializan en las diferentes cadenas comerciales están dentro de los parámetros exigidos por las normas de referencia, por lo que se trata de un producto sin mayor riesgo, en cuanto a aflatoxinas se refiere, para el consumidor vulnerable como son los niños donde pudiera haber afectaciones en cuanto a problemas de retraso del 
crecimiento y vulnerabilidad a enfermedades infecciosas siendo ellos lo más vulnerables ante este problema de contaminación por AF.

Es importante recalcar que se obtuvieron resultados favorables donde el $83.3 \%$ de las muestras presentaron ausencia de AF, así como resaltar que la toxina es capaz de persistir en la matriz alimenticia a pesar de que el hongo toxígeno haya desaparecido. Debido a la composición química de la aflatoxina, los procesos térmicos que reciben las harinas para la producción de cereales infantiles, no elimina por completo la presencia de las micotoxinas debido a su elevada estabilidad térmica, por otro lado al trabajar con temperaturas superiores a las recomendadas en el proceso, se pueden presentar cambios indeseables en la calidad y valor nutricional del producto final, por lo que, los consumidores están expuestos a bajos niveles de aflatoxina dentro de la dieta habitual.

En la actualidad existen técnicas de detección y análisis de aflatoxinas para la regulación de controles reglamentados en laboratorios, así como métodos de control llevados a cabo desde la cosecha para mejorar la resistencia de los cultivos y/o evitar la producción de aflatoxinas, o bien con el uso de controles biológicos combinados con medidas aplicadas después de la cosecha como lo es el secado y almacenamiento con la finalidad de eliminar así las fuentes principales de contaminación. Es difícil modificar los hábitos alimenticios de la población, pero sí se le pueden proporcionar productos sanos mediante un adecuado almacenamiento; es decir, mantener depósitos secos y fríos para evitar el crecimiento de los hongos que las producen.

\section{CONCLUSIÓN}

No se detectó la presencia de aflatoxinas en el $83.3 \%$ de las muestras de los productos analizados y que son comercializados en diferentes cadenas, estando este porcentaje dentro de los límites permitidos por las legislaciones, es decir, el contenido de esta micotoxina está por debajo de $20 \mu \mathrm{g} / \mathrm{kg}$.

A pesar de que los productos cumplen con los límites permitidos por la Norma Oficial Mexicana NOM-247-SSAI/SCFI-2008 y las legislaciones de la Unión Europea, MERCOSUR y Estados Unidos, el $16.7 \%$ de las muestras analizadas contienen $0.83 \mu \mathrm{g} / \mathrm{kg}$ en promedio. Esto constituye un riesgo potencial, ya que estas toxinas con el tiempo van acumulándose en el organismo, pudiéndose manifestar algún tipo de enfermedad hepática en un futuro, incrementándose el riesgo si el alimento es de ingesta continua. 


\section{REFERENCIAS}

1. Abrunhosa, L., Morales, H., Soares, C., Calado, T., Vila-Chã, A. S., Pereira, M. \& Venâncio A. (2014). A Review of Mycotoxins in Food and Feed Products in Portugal and Estimation of Probable Daily Intakes. Food Science and Nutrition, 56, pp. 249-265.

2. Asociación española de fabricantes de cereales. (2011). Cereales de desayuno. 2019, de Asociación española de fabricantes de cereales Sitio web: http://www.asociacioncereales.es/cereales-dedesayuno/historia-y-elaboracion/produccion/

3. Blount, W. P. (1961). “Turkey X Disease,” Journal of British Turkey Federation, Vol. 9, No. 52, pp. 52-61.

4. Brase, S., Encinas, A., Keck, J. \& Nising, C. F. (2009). Chemistry and biology of mycotoxins and related fungal metabolites. Chemical Reviews. 109, pp. 3903-3990.

5. Bogantes, L. P., Bogantes, L. D., \& Bogantes, L. S.. (2004). Aflatoxinas. Acta Médica Costarricense, 46, pp. 174-178.

6. Desjardins, A. E. \& Hohn, T. M. (1997). Mycotoxins in Plant Pathogenesis. Molecular Plant Microbe Interactions. 10, pp. 147-152

7. FAO. (2004). Reglamentos a nivel mundial para las micotoxinas en los alimentos y en las raciones en el año 2003. 2019, de FAO Sitio web: http://www.fao.org/3/y5499s/y5499s00.htm

8.

9. Fokunang, C.N., Tembe, E.A., Tomkins, P., \& Barkwan, S. (2006). Global impact of mycotoxins on human and animal health management. Agriculture 35, pp. 247-253.

10. Frisvad, J. C., Thrane, U., Samson, R. A. \& Pitt, J. I. (2006). Importance mycotoxins and the fungi which produce them. Advances in Food Mycology. 571, pp. 3-31.

11. Gonzalo de Diego Ramos. (2018). Aflatoxinas: el riesgo para la salud oculto en cereales, frutos

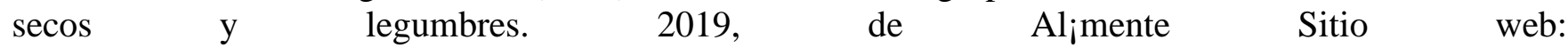
https://www.alimente.elconfidencial.com/nutricion/2018-05-18/aflatoxina-cereal-fruto-secolegumbre_1561894/

12. Organización Mundial de la Salud. (2018). Resumen sobre inocuidad de los alimentos. 2019, de OMS Sitio web: https://www.who.int/foodsafety/FSDigest_Aflatoxins_SP.pdf

13.

14. Khlangwiset, P., Shephard, G. S. \& Wu, F. (2011). Aflatoxins and growth impairment: a review. Critical Reviews in Toxicology. 41, pp. 740-755.

15. Lancaster, M. C., Jenkins, F. P. \& Philp, J. M. (1961). Toxicity associated with certain samples of groundnuts. Nature. 192, pp. 1095-1096.

16. López, G. Park, D., \& Phillips, T. (1999). Contaminación por micotoxinas de alimentos y piensos. 2019, de FAO/OMS/PMA Sitio web: www.bvsde.paho.org/bvstox/e/fulltext/micotox1/micotox1.pdf

17. Mahdavi, R., Nikniaz, L., Arefhosseini, S. R. \& Vahed-Jabbari, M. (2010). Determination of aflatoxin M1 in breast milk samples. Maternal and Child Health Journal. 14, pp. 141-145. 
Moreno, J. (2004). Estudio comparativo de Aspergillus flavus y Aspergillus parasiticus en la producción de aflatoxinas bajo diferentes condiciones de humedad y temperatura. Tesis para obtener el grado de Maestría en Microbiología. FES-Cuautitlán-UNAM

18. Diario Oficial de la Federación. (2002). Norma Oficial Mexicana NOM-188-SSA1-2002, PRODUCTOS Y SERVICIOS. CONTROL DE AFLATOXINAS EN CEREALES PARA CONSUMO HUMANO Y ANIMAL. ESPECIFICACIONES SANITARIAS. 2018, de Secretaría de salud Sitio web: http://www.salud.gob.mx/unidades/cdi/nom/188ssa12.html

19.

20. Organización Mundial de la Salud. (2018). Aflatoxinas. 2019, de OMS Sitio web: https://www.who.int/foodsafety/FSDigest_Aflatoxins_SP.pdf

21. Richard, J. L. (2007). Some major mycotoxins and their mycotoxicose - An overview. International Journal of Food Microbiology. 119, pp. 3-10.

22. Soriano, J. M.. (2007). Micotoxinas en Alimentos. España: Diaz de Santos.

23. Vasanthi, S. \& Bhat, R. V. (1998). Mycotoxins in foods occurrence, health \& economic significance and food control measures. Indian Journal of Medical Research. 108, pp. 212-224.

24. Vega, O. (2012). Hongos micotoxigénicos y aflatoxinas en granos de maíz de diferentes orígenes geográficos de la república mexicana. Tesis para obtener el título de Ingeniero Agrónomo Parasitólogo. Coahuila-México.

25. VICAM L. P.. (1999). Aflatest Instruction manual. 2019, de VICAM Sitio web: www.biotic.com.tw/index.files/GN-MC9508-5\%20(AflaTest).pdf

26. World Health Organization-International Agency for Research on Cancer (WHO-IARC) (1993). Toxins derived from Fusarium moniliforme: fumonisins B1 and B2 and fusarin C. IARC Monographs on the Evaluation of Carcinogenic Risks to Humans. 56, pp. 445-462.

27. Wu, F., Groopman, J. D. \& Pestka, J. J. (2014). Public health impacts of foodborne mycotoxins. Annual Review of Food Science and Technology. 5,pp. 351-372. 